\title{
Simultaneously Diagnosed Autoimmune Hepatitis Type II, Grave's Disease and Congenital Factor VII Deficiency
}

\author{
Vanya Gerova, Ludmila Tankova, Ventsislav Nakov, Gergana Taneva, Radislav Nakov* \\ Clinical Center of Gastroenterology, „Queen Joanna” University Hospital, Sofia, Bulgaria \\ *Corresponding author: radislav.nakov@gmail.com
}

Received May 31, 2015; Revised July 15, 2015; Accepted July 24, 2015

\begin{abstract}
Autoimmune hepatitis (AIH) is progressive, chronic immunologically mediated inflammatory liver disorder that occurs in children and adults of all ages, predominantly in women. AIH can be combined with other autoimmune and non-autoimmune conditions. We present a case of a 32 year-old female with three relatively uncommon diseases - two autoimmune diseases and one inherited, making their combination even more rare.
\end{abstract}

Keywords: autoimmune hepatitis type II, Grave's disease, congenital factor VII deficiency

Cite This Article: Vanya Gerova, Ludmila Tankova, Ventsislav Nakov, Gergana Taneva, and Radislav Nakov, "Simultaneously Diagnosed Autoimmune Hepatitis Type II, Grave’s Disease and Congenital Factor VII Deficiency.” American Journal of Medical Case Reports, vol. 3, no. 9 (2015): 269-271. doi: 10.12691/ajmcr-3-9-1.

\section{Introduction}

Autoimmune hepatitis (AIH) is a chronic immunemediated liver disorder characterised by female preponderance, elevated transaminases and immunoglobulin G levels, seropositivity for autoantibodies and interface hepatitis $[1,2,3]$. AIH can be combined with other autoimmune and non-autoimmune conditions [2,3,4]. Most frequently AIH overlapps with primary biliary cirrhosis (PBC) or primary sclerosing cholangitis (PSC) [3,4]. Associations between AIH and Grave's disease [6], celiac disease [6], multiple sclerosis [7], juvenile idiopathic arthritis [8], alopecia [9] are described in the literature mostly as single case reports or short series.

We present a 32 year-old female with a rare combination of two autoimmune diseases and congenital deficiency of Factor VII.

\section{Case Presentation}

A 32 year-old female was referred to our Clinic with 2 months history of anterior neck swelling, low grade fever, palpitations, diarrhea, progressive weight loss (up to $20 \mathrm{~kg}$ ) and fatigue. During the last week jaundice appeared. She had no previous history of any diseases and alcohol intake. The patient had a history of heavy menorrhagia and frequent bleeding from nose and gums. She did not report bleeding disorders in her family.

Physical examination at admission revealed a toxiclooking woman, wasted, icteric, dehydrated with a slightly enlarged liver and spleen. Her height was $163 \mathrm{~cm}$, body weight - $52 \mathrm{~kg}$, pulse rate - 150/min and blood pressure 130/50 mm Hg.

Table 1. Laboratory Data on Admission

\begin{tabular}{|c|c|c|}
\hline Liver function tests & Results & Reference \\
\hline AST & 869 & $0-41 \mathrm{U} / \mathrm{L}$ \\
\hline ALT & 850 & $0-42 \mathrm{U} / \mathrm{L}$ \\
\hline Total bilirubin & 110 & $0-21 \mu \mathrm{mol} / \mathrm{L}$ \\
\hline Direct bilirubin & 54 & $0-8 \mu \mathrm{mol} / \mathrm{L}$ \\
\hline GGT & 28 & $8-35 \mathrm{mg} / \mathrm{dL}$ \\
\hline ALP & 40 & $40-150 \mathrm{IU}$ \\
\hline Total protein & 81 & $63-84 \mathrm{~g} / \mathrm{L}$ \\
\hline Albumin & 38 & $35-50 \mathrm{~g} / \mathrm{L}$ \\
\hline INR & 3.08 & $0.85-1.25 \mathrm{~s}$ \\
\hline APTT & 24.2 & $22.1-28.1 \mathrm{sec}$ \\
\hline IgG & 28.6 & $7-16 \mathrm{~g} / \mathrm{l}$ \\
\hline $\operatorname{Ig} \mathrm{A}$ & 2.1 & $0.7-4.5 \mathrm{~g} / \mathrm{l}$ \\
\hline $\operatorname{IgM}$ & 1.4 & $0.6-2.5$ \\
\hline Viral markers & Results & Reference \\
\hline IgM anti-HAV & negative & negative \\
\hline HBsAg & negative & negative \\
\hline Anti-HBs & negative & negative \\
\hline Anti-HCV & negative & negative \\
\hline EBV IgM, IgG & negative & negative \\
\hline Cytomegaloviris IgM, IgG & negative & negative \\
\hline Thyroid function test & Results & Reference \\
\hline $\mathrm{TSH}$ & 0.027 & $0.49-4.67 \mathrm{uIU} / \mathrm{mL}$ \\
\hline $\mathrm{fT} 4$ & 2.47 & $0.71-1.85 \mathrm{ng} / \mathrm{dL}$ \\
\hline fT3 & 7.24 & $1.45-3.48 \mathrm{pg} / \mathrm{mL}$ \\
\hline Anti-TPO & 39.3 & $0.00-115.0 \mathrm{IU} / \mathrm{ml}$ \\
\hline Anti-TG & 32.09 & $0.00-115.0 \mathrm{IU} / \mathrm{ml}$ \\
\hline Autoantibodies & Results & Reference \\
\hline ANA & negative & \\
\hline AMA & negative & \\
\hline Anti LKM & positive $1: 160$ & \\
\hline Anti-smooth muscle antibody & negative & \\
\hline
\end{tabular}


Laboratory tests of hepatic function showed elevated aminotransferases (ALAT 850U/L, ASAT 869 U/L), bilirubin total/dir 110/54.6 $\mu \mathrm{mol} / \mathrm{L}$, Immunoglobulin $\mathrm{G}$ (IgG) $28.6 \mathrm{~g} / \mathrm{L}$, total protein $81 \mathrm{mg} / \mathrm{dl}$. Alkaline phosphatase (40 IU/l) and glutamyl transpeptidase were in referent ranges. Coagulation studies showed low protrombin time (PT) - 20\%, high INR of 3.08, normal activated partial thromboplastin time (APTT) and normal platelet count of 412. Direct Coomb's test was negative. Serology for hepatitis A, B, C, Epstein-Barr virus (EBV) and cytomegalovirus were negative. Test for autoimmunity showed the presence of antiliver/kidney microsomal antibodies type 1 (LKM 1) - 1:160; negative anti-mitochondrial (AMA), negative anti-nuclear (ANA) and anti-smooth muscle (ASM) activity. Thyroid function tests were consistent with hyperthyroidism: FT3 7.24 $\mathrm{pg} / \mathrm{mL}$, TF4 $2.47 \mathrm{ng} / \mathrm{dL}$, thyroid stimulating hormone (TSH) $0.027 \mathrm{uIU} / \mathrm{mL}$, Anti-TG $32.09 \mathrm{IU} / \mathrm{ml}$, Anti-TPO $39.3 \mathrm{IU} / \mathrm{ml}$. The laboratory results were consistent with the diagnosis of AIH type II and Grave's disease. The baseline investigations of the patient are shown in Table 1.

Abdominal ultrasound showed slight enlargement of liver and spleen, without evidence of portal hypertension or ascites and normal Doppler investigation. There were no esophageal varices in gastroscopy. High INR was contraindication for liver biopsy. The patient's pretreatment score according to the Revised scoring system for diagnosis of $\mathrm{AIH}$ was 17, indicating 'definite' $\mathrm{AIH}$ with $95 \%$ sensitivity, 97\% specificity and 94\% diagnostic accuracy [10].

Treatment was initiated with Methimazole (MMI) 10mg daily, Prednisolone $30 \mathrm{mg} /$ day and Azathioprine (50 $\mathrm{mg} /$ day). Aminotransferases and bilirubin values returned to normal range after 2 weeks of treatment (ALAT 38U/L, ASAT $32 \mathrm{U} / \mathrm{L}$, bilirubin total/dir 21/7.5 $\mu \mathrm{mol} / \mathrm{L})$. Prednisolone was gradually decreased and after 3 months of therapy was switched to the local steroid - Budesonide $9 \mathrm{mg} / \mathrm{per}$ day. The last medicine was stopped after gradually tapering during 6 months and the patient was treated with Azathioprine alone.

The patient was under regular follow-up during which liver function tests were kept in normal range except persistence of high level of INR, inadequate to the degree of liver damage. She was referred to Department of Haematology and diagnosed with congenital factor VII deficiency with $5 \%$ level of normal factor VII.

The patient was kept euthyroid and at the end of 12 months of treatment, MMI was discontinued. After 4 years of treatment, the patient continues to have normal liver tests as well as normal thyroid function.

\section{Discussion}

AIH is progressive, chronic immunologically mediated inflammatory liver disorder that occurs in children and adults of all ages, predominantly in women [1,2,3]. The disease is subclassified into two major types: AIH type 1 (AIH-1) positive for anti-nuclear (ANA) and/or antismooth muscle antibodies (SMAs) and AIH type 2 (AIH2) defined by the presence of anti-liver kidney microsomal antibody type 1 (LKM-1) and/or anti-liver cytosol type 1 (LC-1) autoantibodies [1,3,5]. AIH-2 more frequently presents at a younger age, and commonly with IgA deficiency, has an acute or severe course and advanced histological lesions at presentation; however, duration of symptoms before diagnosis, clinical signs, family history of autoimmunity, presence of associated autoimmune disorders, response to treatment, and long-term prognosis are similar in the two group [2]. Autoimmune hepatitis responds to immunosuppressive treatment, and treatment should be initiated promptly to avoid progression of disease $[1,11,12]$.

The diagnosis of AIH in our patient is made on the basis of the clinical, biochemical and immunological studies, after exclusion of other liver diseases -viral, toxic, metabolic and PBC. The presence of positive LKM-1 antibodies together with negative AMA, ANA and SMA excludes PBC and supports the diagnosis of AIH type 2.

The interface hepatitis is characteristic histologic finding in AIH $[1,2,10]$. However, liver biopsy was not performed in this patient due to persistent high levels of INR most probably not related to the liver damages. As a cause of the coagulation disorder was found Factor VII deficiency, a rare congenital coagulopathy. Factor VII is one of the vitamin K-dependent coagulation factors synthesized in the liver [13]. There are more than 100 mutations in factor VII gene, located on 13th chromosome mostly missense mutations [13,14]. Most likely, Factor VII deficiency in our patient is congenital and pre-existing liver disease, because of the presence of history of frequent bleeding from nose and gums since childhood, and later history of severe menorrhagia.

$\mathrm{AIH}$ can be associated with various autoimmune diseases - hepatic (PBC, PSC) or extrahepatic. The autoimmune thyroid disease most commonly combines with AIH. In our patient AIH is combined with Grave's disease. Graves' disease is an autoimmune disease characterized by the presence of activating autoantibodies against thyroid stimulating hormone (TSH) receptor that results in the overproduction of thyroid hormones (hyperthyroidism). The disease may affect anyone and is more frequent among women below the age of 40 . The clinical features are largely diverse and in most of the cases liver function abnormalities are included [15]. The thyroid disease of our patient was confirmed on the basis of clinical and laboratory data for hyperthyroidism.

The associated autoimmune disease may precede the appearance of AIH or occur after the diagnosis of the liver disease is confirmed. In our patient the two autoimmune diseases are diagnosed at the same time after a period of probably subclinical course. More pronounced, however, were the clinical signs of hyperthyroidism. Both diseases coexist; thyroid disease does not worsen the clinical course of AIH and vice versa. Both, AIH and Grave's disease have responded well to treatment with no evidence of side effects, laboratory parameters for liver and thyroid functions have been within normal range. For the reason that our patient feels well, she refused a liver biopsy after pre-treatment with recombinant factor and fresh frozen plasma substitution.

\section{Conclusion}

We present a case with three relatively uncommon diseases - two autoimmune diseases and one inherited, making their combination even rarer. The findings of this 
case indicate that simultaneously appeared autoimmune diseases do not alter their clinical course or severity. Due to the possible combination routine screening for autoimmune thyroid disease in patients with $\mathrm{AIH}$ is reasonable.

\section{References}

[1] Krawitt, E.L., "Autoimmune hepatitis," N Engl J Med, 354 (1), 5466, Jan 2006

[2] Mieli-Vergani, G. and Vergani, D., "Autoimmune Hepatitis in Children: What is Different from Adult AIH?," Semin Liver Dis, 29 (3), 297-306, Aug 2009.

[3] Gatselis, N.K., Zachou, K., Koukoulis, G. and Dalekos, G. "Autoimmune hepatitis, one disease with many faces: Etiopathogenetic, clinico-laboratory and histological Characteristics," World J Gastroenterol, 21 (1), 60-83, Jan 2015

[4] Teufel, A., Weinmann, A., Kahaly, G.J., Centner, C., Piendl, A., Worns, M., Lohse, A.W., Galle, P.R. and Kanzler, S., "Concurrent autoimmune diseases in patients with autoimmune hepatitis," $J$ Clin Gastroenterol, 44 (3), 208-13, Mar 2010.

[5] Jhee, J.H., Kim, H.J., Kang, W., Kim. S. and Kim, D.Y., "A Case of Autoimmune Hepatitis Combined with Graves' Disease," Korean J Gastroenterol, 65 (1), 48-51, Jan 2015.

[6] Milic, S., Micolasevic, I., Bulic, Z., Giljaca, V. and Stimac, D., "Celiac disease associated with autoimmune hepatitis and autoimmune hyperthyroidism," Med Glas(Zenica), 10 (2), 408-10, Aug 2013.
[7] Nadhem, O.N., Janabi, M.A., Omer, A.R. and Wan, B. "Autoimmune hepatitis with multiple sclerosis and graves disease: coincidence or association?," Case Rep Gastroenterol, 8 (2), 31923, Oct 2014.

[8] Nobili, V., Devoto, R., Comparcola, D., Cortis, E., Sartorelli, M.R and Marcellini, M., "Juvenile idiopathic arthritis associated with autoimmune hepatitis type 2," Ann Rheum Dis, 64 (1), 157-158, Jan 2005.

[9] Pereira, T.C., Bellomo-Brandão, M.A. and Hessel, G., "Alopecia in autoimmune hepatitis:Association with zinc deficiency," Annals of Gastroenterology, 23 (1), 71-73, Jan 2010.

[10] Alvarez, F., Berg, P.A., Bianchi, F.B. et al., "International autoimmune hepatitis group report: review of criteria for diagnosis of autoimmune hepatitis," Journal of Hepatology, 31 (5), 929-938, Nov 1999.

[11] Fallatah, H.I. and Akbar, H.O. "Autoimmune Hepatitis as a Unique Form of an Autoimmune Liver Disease: Immunological Aspects and Clinical Overview," Hindawi Publishing Corporation Autoimmune Diseases, Article ID 312817, 17 pages, 2012.

[12] Lapecorellia, M. and Mariani, G. "Factor VII deficiency: defining the clinical picture and optimizing therapeutic options," Haemophilia, 14 (6), 1170-1175, Nov 2008.

[13] Bakkali, H., Massou, S., Labrini, F., Mounir, K., Bensghir, M., Azendour, H., Balkhi, H. and Haimeur, C. "Congenital deficiency in factor VII of the coagulation and the anesthesia," Journal of Anesthesiology, 1 (3), 24-26, Nov 2013.

[14] Zachou, K., Muratori, P., Koukoulis, G.K., Granito, A., Gatselis, N., Fabbri, A., Dalekos, G.N. and Muratori, L. "Review article: autoimmune hepatitis - current management and challenges," Aliment Pharmacol Ther, 38 (8), 887-913, Oct 2013.

[15] Bartalena, L. "Diagnosis and management of Graves disease: a global overview," Nat Rev Endocrinol, 9 (12), 724-734, Dec 2013. 